\title{
Contact Screening and Isoniazid Preventive Therapy Initiation for Under-Five Children among Pulmonary Tuberculosis-Positive Patients in Bahir Dar Special Zone, Northwest Ethiopia: A Cross- Sectional Study
}

\author{
Netsanet Fentahun $\left(\mathbb{D},{ }^{1}\right.$ Yosef Wasihun $\left(\mathbb{D},{ }^{1}\right.$ Abebe Mamo, ${ }^{2}$ and Lakew Abebe Gebretsadik ${ }^{2}$ \\ ${ }^{1}$ School of Public Health, College of Medicine and Health Science, Bahir Dar University, Bahir Dar, Ethiopia \\ ${ }^{2}$ Department of Health, Behavior and Society, Institutes of Health, Jimma University, Jimma, Ethiopia \\ Correspondence should be addressed to Netsanet Fentahun; netsanet.fentahun@bdu.edu.et
}

Received 19 February 2020; Revised 6 May 2020; Accepted 16 May 2020; Published 6 June 2020

Academic Editor: José R. Lapa e Silva

Copyright (c) 2020 Netsanet Fentahun et al. This is an open access article distributed under the Creative Commons Attribution License, which permits unrestricted use, distribution, and reproduction in any medium, provided the original work is properly cited.

\begin{abstract}
Background. Children are highly susceptible to Mycobacterium tuberculosis infection, and about $70 \%$ of children living in the same households with pulmonary tuberculosis-positive patients will become infected. However, pulmonary positive tuberculosis is a common phenomenon and the implementation of the recommended contact screening and initiation of isoniazid preventive therapy is very low. Therefore, this study is aimed at assessing contact screening practice and initiation of isoniazid preventive therapy of under-five children among pulmonary tuberculosis-positive patients in Bahir Dar, northwest Ethiopia. Methods. A facility-based cross-sectional study was conducted from March 1 to 30, 2016. A total of 267 pulmonary tuberculosis-positive patients were included in this study. To identify independent predictors of contact screening and isoniazid preventive therapy initiation, we performed multivariable logistic regression analyses using SPSS version 20 with CI of $95 \%$ at $p$ value $<0.05$. Results. A total of 230 (90.2\%) pulmonary tuberculosis-positive patients had single contacts with their under-five children. One hundred nine $(64.8 \%)$ children were screened. From those screened, 11 (7.4\%) developed tuberculosis disease and started antituberculosis treatment. Forty-four (31.9\%) children started isoniazid preventive therapy. Sex of the participants, place of service delivery, relationship with contacts, HIV status, and attitude of PTB+ cases were significant predictors of contact screening $(p<.05)$. Participant's knowledge, attitude of participants, and relationship of the child with participant were significant predictors of isoniazid preventive therapy initiation $(p<0.05)$. Conclusion. Contact screening practice and isoniazid preventive therapy initiation of children under the age of 5 in Bahir Dar zone were very low. Intimate family contact with pulmonary tuberculosis-positive patients, place of service delivery, and attitude towards screening are the key factors of contact screening. Participant's knowledge, attitude of participants, and relationship of the child with participant are the key factors of isoniazid preventive therapy initiation. Therefore, household contact screening and isoniazid preventive therapy initiation should be paid attention to reduce transmission.
\end{abstract}

\section{Introduction}

Tuberculosis (TB) is a chronic necrotizing disease caused by Mycobacterium tuberculosis complex. The species commonly involved are M. tuberculosis, M. bovis, M. africanum, and M. microti [1]. Worldwide, an estimated 10 million people fall ill with TB and about 1.5 million cases died in
2018. From these 10 million cases, about 1.1 million $(11 \%)$ occurred in children (0-14 years) and there were 205,000 child deaths due to TB in 2018 [2]. The World Health Organization (WHO) reported that a number of new TB cases occurred in Southeast Asia (44\%), Africa (24\%), and Western Pacific (18\%) globally in 2018 [2]. Almost $90 \%$ of cases each year are in 30 high TB burden 
countries, and eight countries account for two-thirds of the total [3].

In Ethiopia, TB is one of the leading infectious diseases, and in 2014/2015, 135,831 TB cases (all forms) were reported; of these, $35 \%$ were bacteriologically confirmed pulmonary TB cases [4].

TB ranks among the top causes of childhood morbidity and mortality in developing countries but neglected for years in children [5]. Children living in close contact with PTB+ are at high risk of $\mathrm{TB}$ infection. One cough can produce 3,000 and a sneeze up to a million droplet nuclei in which the infectious dose of TB is 1 to 10 bacilli [6].

Contact screening and providing treatment are important and a simple strategy to implement at the primary health care setting [7]. Various studies demonstrated screening as an effective strategy in early identification of children eligible for isoniazid preventive therapy (IPT) and preventing susceptible children from developing the disease who had household contacts with $\mathrm{PTB}+$ patients [8-10]. WHO and CDC reported that IPT is effective for about $93 \%$ of under-five children and 59\% among children aged 15 years or younger and IPT is recommended in a daily basis for at least 6 months [11-13].

Rates for the initiation of IPT in eligible children ranging from $1.3 \%$ to $26 \%$ are reported in settings where tuberculosis is endemic [14]. High-burden countries have reported extremely poor compliance with screening and initiation of IPT because of limited awareness of its benefits and inability to perform screening tests by health care providers. Interruption of INH supply and distance from health facilities affect uptake of the service in different settings [15].

Contact tracing, investigation, and prophylaxis of childhood contacts of adult TB cases are widely recommended but rarely practiced in developing countries [16]. Ethiopia is also one of the 22 high-burden countries for TB, and childhood TB is still a major cause of hospital admission and death [17]. In 2014, of the new cases notified in Ethiopia, fourteen percent were pediatric TB cases. Even this could be an underestimate due to difficulty in confirmation of diagnosis of TB in children [18]. Ethiopia has accepted and implemented the WHO's recommendation of a six-month course of IPT as one strategy for prevention, care, and control of TB [19]. Ethiopia also developed a national TB prevention and treatment guideline which emphasizes on contact screening and IPT for under-five children who have a history of contact with $\mathrm{PTB}+$ patients. Clinical assessment alone is conducted to decide whether the contact is eligible for IPT initiation or not. But for symptomatic contacts, further diagnosis like chest radiograph and ESR is conducted to rule out active TB $[8,20]$.

However, it has been evident that the implementation of the recommended contact screening is very low and IPT initiation has been largely ignored in Ethiopia [8, 15]. In addition, there is limited information regarding suboptimal contact screening and noninitiation of IPT. These indicated that there is a huge gap in implementing the recommended strategies. Hence, identifying and understanding the factors that affect the implementation of contact screening and IPT initiation are crucial to address the challenges. Therefore, the objective of this study was to assess the contact screening and INH prophylaxis practices of under-five children among $\mathrm{PTB}+$ patients in Bahir Dar special zone, northwest Ethiopia.

\section{Methods and Materials}

2.1. Study Design and Settings. This study used a facilitybased cross-sectional study design to assess household contact screening and INH prophylaxis of under-five children who had contact with PTB+. We conducted this study in Bahir Dar special administration zone, with 221,991 predominantly urban populations, Amhara regional state of Ethiopia. It is located around $565 \mathrm{~km}$ northwest of Addis Ababa, the capital of Ethiopia. In this zone, there were around 989 TB patients of all forms and 460 were $\mathrm{PTB}+$ patients. The special zone has 18 governmental health facilities ( 4 hospitals and 14 health centers), which were giving directly observed treatment (DOT) services in the first quarter of 2016.

2.2. Study Participants and Procedures. The study population was all $\mathrm{PTB}+$ patients who had under-five children and registered their household contacts during November 2015March 2016. These participants were interviewed during DOTS program at health facilities from March 1 to 30, 2016. As per guidelines [8], all diagnosed PTB+ patients are expected to be recorded in the contact investigation register. So, we used the data routinely recorded in the contact investigation register at all health facilities (4 hospitals and 14 health centers) providing DOT services in the study areas. From these health facilities, there were around 460 PTB+ patients, and 267 of them were listed on TB treatment registration book as $\mathrm{PTB}+$ patients who had under-five children and we included all 267 of them as census. Every day, we gathered all $\mathrm{PTB}+$ patients in the morning and selected those who had under-five children at home for interview. Patients' TB treatment cards were used for identification of those PTB+ patients. During data collection, those children who were screened and started IPT and anti-TB treatment were cross-checked from IPT and anti-TB treatment registration books, respectively.

The Ethiopian National TB Program recommends the following groups of persons to be given priority for identification through contact tracing and undergo clinical evaluation: symptomatic contact of the index patient, contacts who are living with HIV, contacts who are under-five children, and contact of an index case with presumed/confirmed DR-TB. Contact tracing is initiated by the TB focal person as soon as the index case is registered to receive TB treatment. The TB focal person communicates with the health extension workers to conduct identification of contacts at the household/community level, do symptom-based TB screening, and refer those who require detailed evaluation and investigation at the health facility level. The initial identification and evaluation of identified contacts for active TB are conducted using symptom-based $\mathrm{TB}$ screening questions and may be conducted at the community level by health extension workers and/or at a health facility by trained health care workers. 


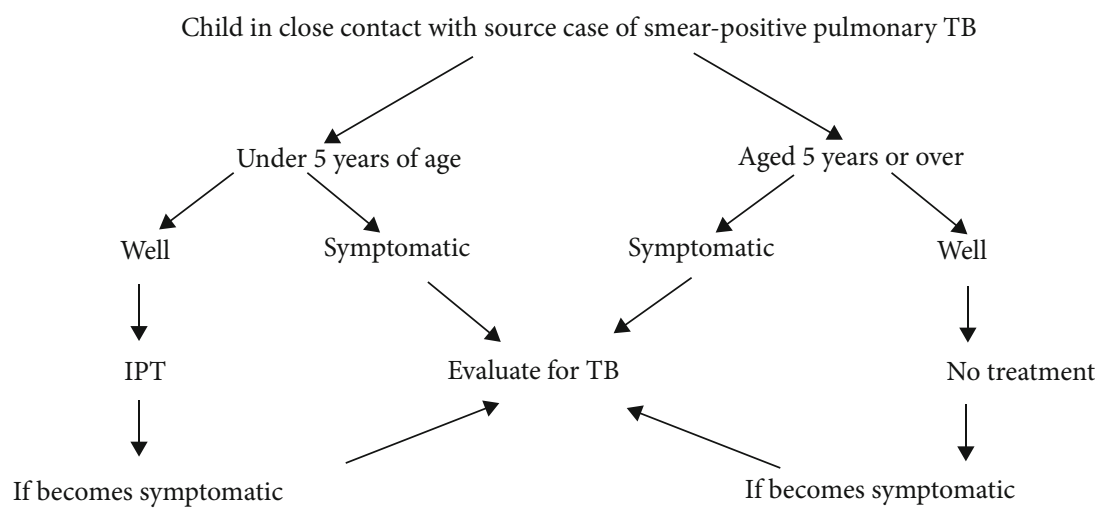

FIGURE 1: Approach contact management of children in Ethiopia.

In Ethiopia, we have two modalities for TB contact tracing. The first modality is contact tracing at the community level: as part of the community TB care package, health extension workers are practicing household contact tracing during routine home visits and upon receiving the request from the catchment health facility. The health extension workers are preferred to identify those at high risk of developing $\mathrm{TB}$ and symptomatic ones and bring them to the health facility for clinical evaluation and subsequent management. The second modality is contact tracing at the health facility level: the health care worker at the TB clinic should initiate contact tracing and investigation for close/household contacts upon registering a new index TB patient for treatment (Figure 1).

2.3. Data Collection and Measurements. PTB+ patients with child contacts were interviewed in Amharic (local language) using a pretested, structured, and interviewer-administered questionnaire. The questionnaire is developed by reviewing different literatures [21-24] and prepared in English first and translated into the local language. The questionnaire included sociodemographic variables, knowledge, and attitude of PTB+ patients on tuberculosis, contact screening, and INH prophylaxis and health worker-related questions. The questionnaire was pretested in Merawi district which is found $32 \mathrm{~km}$ far from the study area. The data were collected by four college completed BSc nurses and one health officer as a supervisor. Training was given for data collectors about the data collection tool, how to collect data, and taking consent to have common understanding.

The knowledge question was assessed using different items including sign and symptoms, cause, mode of transmission, risk groups, prevention mechanism, and treatment of tuberculosis. Study participants were interviewed on all mentioned dimensions regarding tuberculosis with "yes or no" questions, and finally, a composite measure for each aspect was created out of 15 and this weighted score was used for analysis as continuous variable.

The attitude part of the questions consisted of five items in five-point Likert scale. The scores of individuals for each item were summed up and ranged from 5 minimum to 25 maximum total score after reverse coding for negatively worded items, and this was treated as continuous variable for analysis. Reliability test was checked using Cronbach's alpha and the results were 0.87 and 0.74 , respectively.

Contact screening was assessed using two items with the responses of "Yes or No" type like "have you ever heard or been informed about contact screening"? Those who responded "Yes" were considered as eligible for the next question and were asked "have your under-five child/children been screened for TB"? Those who responded "Yes" were considered as they were practicing contact screening. Finally, children who were screened and found free from TB were identified and considered as eligible for IPT and assessed using an item, "did your child/children start INH prophylaxis"? Those who responded "Yes" were considered as the children were starting IPT.

2.4. Data Analysis. Data were coded and entered to a computer using EpiData software version 3.1 (EpiData Association, Odense, Denmark) and exported to SPSS program version 20.0 for further analysis. The descriptive result was presented using frequency and proportions for all variables, and we performed bivariate regression analysis to determine association between factors of contact screening and IPT initiation at $95 \%$ confidence interval and $p$ value of $<0.25$. We performed multivariate logistic regressions, and variables with $p$ value $<0.05$ are considered to identify the independent predictors of contact screening and IPT initiation. Goodness of fit of the final models was checked using Hosmer and Lemeshow test of goodness fit for contact screening and IPT, and the results were 0.86 and 0.26 , respectively.

\section{Result}

3.1. Sociodemographic Characteristics. Overall, 267 PTB+ patients who had household contacts were registered between November 2015 and March 2016. A total of 255 $\mathrm{PTB}+$ patients were interviewed from March 1 to 30, 2016, with a response rate of $95.5 \%$. From the interviewed PTB+ patients, $131(51.4 \%)$ were males, $153(60 \%)$ of them were parents (father or mother) of the contacts, and more than half of the respondents 134 (52.5\%) were married. Other sociodemographic characteristics of respondents and related factors are given in Table 1. 
TABLE 1: Sociodemographic and related profile of PTB+ patients in Bahir Dar special zone, northwest Ethiopia, March $2016(N=255)$.

\begin{tabular}{|c|c|c|c|}
\hline Variables & Categories & Frequency & Percent \\
\hline \multirow{5}{*}{ Age in years } & $<20$ & 36 & 14.1 \\
\hline & $20-29$ & 89 & 34.9 \\
\hline & $30-39$ & 84 & 32.9 \\
\hline & $40-49$ & 32 & 12.5 \\
\hline & $>49$ & 14 & 5.5 \\
\hline \multirow{2}{*}{ Sex } & Male & 131 & 51.4 \\
\hline & Female & 124 & 48.6 \\
\hline \multirow{2}{*}{ Religion } & Christian & 239 & 93.7 \\
\hline & Muslim & 16 & 6.3 \\
\hline \multirow{2}{*}{ Residence } & Urban & 168 & 65.9 \\
\hline & Rural & 87 & 34.1 \\
\hline \multirow{4}{*}{ Marital status } & Married & 134 & 52.5 \\
\hline & Unmarried & 101 & 39.6 \\
\hline & Divorced & 7 & 2.7 \\
\hline & Widowed & 13 & 5.1 \\
\hline \multirow{4}{*}{ Educational status } & $\begin{array}{l}\text { No formal } \\
\text { education }\end{array}$ & 75 & 29.4 \\
\hline & $\begin{array}{l}\text { Primary school } \\
\text { educated }\end{array}$ & 100 & 39.2 \\
\hline & $\begin{array}{c}\text { Secondary school } \\
\text { educated }\end{array}$ & 71 & 27.8 \\
\hline & $\begin{array}{l}\text { College/university } \\
\text { educated }\end{array}$ & 9 & 3.5 \\
\hline \multirow{8}{*}{ Occupation } & Farmer & 50 & 19.6 \\
\hline & Merchant & 41 & 16.1 \\
\hline & Student & 36 & 14.1 \\
\hline & Housewife & 32 & 12.5 \\
\hline & Daily laborer & 27 & 10.6 \\
\hline & $\begin{array}{l}\text { Government } \\
\text { employee }\end{array}$ & 20 & 7.8 \\
\hline & No work & 26 & 10.2 \\
\hline & Driver & 23 & 9 \\
\hline \multirow{3}{*}{ Household income } & $<500$ eth birr & 106 & 41.6 \\
\hline & 500-1000 eth birr & 83 & 32.5 \\
\hline & $>1,000$ eth birr & 66 & 25.9 \\
\hline \multirow{2}{*}{$\begin{array}{l}\text { Relationship of the } \\
\text { index cases with } \\
\text { contacts }\end{array}$} & Parents & 153 & 60 \\
\hline & Siblings & 102 & 40 \\
\hline
\end{tabular}

3.2. Health Information Related to Contact Screening and IPT Initiation. From the 255 interviewed PTB+ patients, $230(90.2 \%)$ of them informed having under-five contacts (1 under-five contact/PTB+ patient), for a total of 230 under-five children evaluated. Of these 230 under-five children, 149 (64.8\%) of them were screened for active TB and $11(7.4 \%)$ were diagnosed with TB disease and started antiTB treatment. One hundred thirty-eight child contacts who were diagnosed as free from TB disease were considered as
TABLE 2: Health information related to contact screening and IPT initiation of PTB+ patients in Bahir Dar special zone, northwest Ethiopia, March $2016(N=255)$.

\begin{tabular}{lccc}
\hline Variable & Category & Frequency & Percent \\
\hline Informed about contact & Yes & 230 & 90.2 \\
screening & No & 25 & 9.8 \\
Practice contact screening & Yes & 149 & 64.8 \\
$(N=230)$ & No & 81 & 35.2 \\
Outcome of contact screening & Positive & 11 & 7.4 \\
& Negative & 138 & 92.6 \\
Informed about the presence & Yes & 82 & 32.2 \\
of IPT & No & 173 & 67.8 \\
Started to use IPT $(N=138)$ & Yes & 44 & 31.9 \\
& No & 94 & 68.1 \\
HIV status $(N=230)$ & Positive & 34 & 14.8 \\
& Negative & 196 & 85.2 \\
Place of service provision & Health & 190 & 82.6 \\
$(N=230)$ & center & 40 & 17.4 \\
\hline
\end{tabular}

IPT: isoniazid preventive therapy; PTB+: smear-positive pulmonary tuberculosis; HIV: human immuno virus.

eligible for IPT; of whom, 44 (31.9\%) started IPT. The mean score of knowledge and attitude was $9.74(\mathrm{SD} \pm 3.94)$ and 17.81 ( $\mathrm{SD} \pm 2.59$ ), respectively. Contact screening, initiation of IPT, and other related factors are given in Table 2.

3.3. Factors Associated with Household Contact Screening of Under-Five Children. After controlling for possible confounding factors through multiple logistic regressions, place of service provided, relationship of the index case with the contact, and attitude of respondents were independent predictors of contact screening. Females who had contact with children were five times more likely to bring their underfive children for contact screening than males $(\mathrm{AOR}=5.3$, 95\% CI $(1.2,23.2))$. Participants who received the services from a hospital were 7 times more likely to bring their under-five children for contact screening as compared to those participants who received the services from a health center $(\mathrm{AOR}=6.5,95 \% \mathrm{CI}(1.2,41.8))$. Parents who had a contact with children were 15 times more likely to bring their under-five children for contact screening as compared to siblings (brothers, sisters, and other relatives) (AOR $=14.8,95 \%$ CI $(3.2,69.7))$. HIV-positive TB patients were 19 times more likely to bring their under-five children for contact screening as compared to HIV-negative TB patients (AOR $=19,95 \%$ CI $(2.1,16.87))$. A unit increase in total score of attitude of the participants in the odds of household contact screening was increased by $2.8(\mathrm{AOR}=2.8,95 \% \mathrm{CI}(1.5,5.2))$ (Table 3$)$.

3.4. Factors Associated with Initiation of IPT for Under-Five Children. Multiple logistic regression analysis was performed to identify independent predictors of IPT initiation. Children who have parents as index case were twenty times more likely to start IPT as compared to children who had siblings $(\mathrm{AOR}=20.0,95 \%$ CI $(2.4,168.1))$. A unit increases of 
TABLE 3: Factors associated with household contact screening of under-five children, about contact screening in Bahir Dar special zone, northwest Ethiopia, March $2016(N=230)$.

\begin{tabular}{|c|c|c|c|c|c|}
\hline \multirow{2}{*}{ Variables } & & \multicolumn{2}{|c|}{ Contact screening } & \multirow{2}{*}{ COR $(95 \%$ CI $)$} & \multirow{2}{*}{$\mathrm{AOR}(95 \% \mathrm{CI})$} \\
\hline & & Yes & No & & \\
\hline \multirow{2}{*}{ Sex } & Male & $69(46.3 \%)$ & $50(61.7 \%)$ & 1 & 1 \\
\hline & Female & $80(53.7 \%)$ & $31(38.3 \%)$ & $1.9(1.1,3.2)$ & $5.3(1.2,23.2) *$ \\
\hline \multirow{2}{*}{ Residence } & Rural & $29(19.5 \%)$ & $40(49.4 \%)$ & 1 & 1 \\
\hline & Urban & $120(80.5 \%)$ & $41(50.6 \%)$ & $4.1(2.2,7.3)$ & $1.02(0.2,5.5)$ \\
\hline \multirow{2}{*}{ Health facility } & Health center & $114(76.5 \%)$ & $76(93.8 \%)$ & 1 & 1 \\
\hline & Hospital & $35(23.5 \%)$ & $5(6.7 \%)$ & $4.7(1.8,12.4)$ & $6.5(1.2,41.8) *$ \\
\hline \multirow{2}{*}{ HIV status } & Negative & $119(79.9 \%)$ & $77(95.1)$ & 1 & 1 \\
\hline & Positive & $30(20.1 \%)$ & $4(4.9 \%)$ & $4.9(1.6,14.3)$ & $19(2.1,16.87) * *$ \\
\hline \multirow{2}{*}{ Relationship of the child with participant } & Parents & $107(71.8 \%)$ & $34(42 \%)$ & $3.5(2,6.2)$ & $14.8(3.2,69.7) * *$ \\
\hline & Sibling ${ }^{\#}$ & $42(28.2 \%)$ & $47(58 \%)$ & 1 & 1 \\
\hline$* *^{\#}$ Knowledge score & & & & $1.7(1.5,1.9)$ & $0.9(0.6,1.3)$ \\
\hline$* *^{\#}$ Attitude score & & & & $3.1(2.3,4.2)$ & $2.8(1.5,5.2) * *$ \\
\hline
\end{tabular}

*Continuous variables. AOR: adjusted odds ratio; COR: crude odds ratio; HIV: human immuno virus. "Sibling: brothers, sisters, and relatives.

TABLE 4: Factors associated with initiation of IPT of under-five children, in Bahir Dar special zone, northwest Ethiopia, March $2016(N=138)$.

\begin{tabular}{|c|c|c|c|c|c|}
\hline \multirow{2}{*}{ Variable } & & \multicolumn{2}{|c|}{ IPT prophylaxis } & \multirow{2}{*}{ COR with 95\% CI } & \multirow{2}{*}{ AOR with $95 \% \mathrm{CI}$} \\
\hline & & Started & Not started & & \\
\hline \multirow{2}{*}{ Relationship of the case with contacts } & Siblings & $2(4.5 \%)$ & $37(39.4 \%)$ & 1 & 1 \\
\hline & Parent & $42(95.5 \%)$ & $57(60.6 \%)$ & $13.6(3.1,59.7)$ & $20.0(2.4,168.1)$ \\
\hline \multirow{3}{*}{ Age of the child } & $<1$ year & $3(6.8 \%)$ & $40(42.6 \%)$ & $0.07(0.02,0.3)$ & $1.9(0.3,11.2)$ \\
\hline & 1-2 years & $11(25 \%)$ & $26(27.7 \%)$ & $0.4(0.2,1.0)$ & $1.8(0.3,11.2)$ \\
\hline & $>2$ years & $30(68.2 \%)$ & $28(29.8 \%)$ & 1 & 1 \\
\hline \multirow{2}{*}{ HIV status } & Negative & $25(56.8 \%)$ & $86(91.5 \%)$ & 1 & 1 \\
\hline & Positive & $19(43.2 \%)$ & $8(8.5 \%)$ & $8.2(3.2,20.9)$ & $0.4(0.5,1.5)$ \\
\hline *Attitude & & $\beta=1.230$ & & $6.8(3.2,14.5)$ & $3.4(1.1,10.2)$ \\
\hline$*$ Knowledge & & $\beta=1.065$ & & $3.1(1.9,4.9)$ & $2.9(1.6,5.2)$ \\
\hline
\end{tabular}

*Continuous variables. AOR: adjusted odds ratio; COR: crude odds ratio; IPT: isoniazid preventive therapy.

attitude of participants; the odds of IPT initiation is increased by 3.4 (AOR, 95\% CI $3.4(1.1,10.2)$ ). A unit increases of knowledge of the participants; the odds of starting IPT initiation is increased by 2.9 (AOR, 95\% CI $2.9(1.6,5.2)$ ) (Table 4).

\section{Discussion}

This study provides insight into the operation of household contact screening and IPT initiation of under-five children in northwest Ethiopia. About $64.8 \%$ of child contacts underwent screening for TB in our study, and other studies from Vietnam [25], Malawi [26], and Thailand [27] reported much less screening rates (6-52\%), while a study in Western Cape Province of South Africa reported much higher screening rates (91\%) [28]. The possible explanation for these differences could be due to the lack of an extensive awareness campaign about $\mathrm{TB}$ and its treatment, passive contact screening method, and lack of follow-up of the index cases to bring their under-five children to health facilities by health workers.
In addition to contact screening, this study also found that only $31.9 \%$ of children were started with IPT. This finding is lower than a study reported in Indonesia which reported $40 \%$ of IPT initiation [29], much lower than a study in India (56.4\%) [30], but higher than other study in India (19\%) of IPT initiation [21]. Inaccessibility of health facilities, isoniazid stock-outs, and risk perception of participants to start IPT without any sign and symptoms could be the main justifications for low initiation of IPT in our study.

In this study, contact screening and initiation of IPT were more likely among child contacts where index case is parents than siblings. This is consistent with the study findings from Vietnam [25] and Timor Leste [31]. In India, children living with parents were more likely to have initiated on IPT than other household members [32]. This study implies that parents are more concerned for their children's health and well-being and the bond between parents and small children are stronger than other household members. These reasons have also been reported in a study from Thailand [27].

This study also found that household contact screening of under-five children was more likely among hospital users 
than health center users. Studies from Thailand and Malawi reported that health center-based contact screening was poorly used because different types of laboratory services and tests were not available in the health centers, so the hospital is identified as a better option [27, 33].

This study showed that HIV-positive patients had significant association with contact screening unlike a previous study [22]. This could be because HIV-positive patients had regular visits to a health facility for antiretroviral therapy follow-up which might in turn made them know the benefit of early contact screening than HIV-uninfected patients.

Attitude of tuberculosis patients towards household contact screening without signs and symptoms and starting IPT in eligible under-five children had significant association with contact screening as well as IPT utilization. This finding was coherent with a study in India which revealed that most TB patients like to use screening and initiate IPT and they believed that the use of IPT has contributed to a higher reduction of acquiring tuberculosis [33]. This implies that if people believe in positive outcome, they accept it without trying to prove that the treatment is working or not.

According to this study, we found that knowledge was a major influencing factor for contact screening of under-five children. Per a unit increase in total score of knowledge on preventive mechanisms of TB, the odds of IPT initiation is also increased. A study in Ethiopia explained that 79.3\% responded transmission of $\mathrm{TB}$ would be preventable and $80 \%$ knew that TB can be transmitted from a patient to another person [18]. Different studies justified that highburden countries have reported extremely poor compliance with initiation of IPT because of limited awareness of its benefits and inability to perform prerequisite screening tests.

Rates for the initiation of IPT in eligible children ranging from 1.3 to $26 \%$ have been reported in settings where tuberculosis is endemic [14, 22]. As explained in a health belief model, when the barriers of health action outweigh the benefits in the minds of a given person, the likelihood of taking action decreases [33]. Therefore, certain types of barriers are more or less important for particular cultures or norms. Thus, it is not only knowledge but also attitude and other factors which could be special factors associated with the initiation of IPT among participants. As we conducted this study, there were a few limitations, which relate to the use of selfreported questions on knowledge of patients on tuberculosis, contact screening, and INH prophylaxis, which could lead to social desirability biases. However, as these questions were validated in a similar population, it is expected that this problem is minimal. Again, this study is conducted in one zone of the Amhara region. Whether the findings from this administrative zone are representative of contact screening and IPT of the whole region is unknown. But this special administrative zone has relatively higher number of population than other zones.

\section{Conclusion}

Household contact screening of under-five children and initiation of IPT in this study were very low. Sex of the participants, place of service delivery, relationship with contacts,
HIV status, and attitude of PTB+ cases are the key factors for contact screening. Knowledge of participants, attitude of participants, and relationship with contacts are the key factors of initiation of IPT. The regional health bureau and health facilities should pay attention on contact screening and IPT initiation to narrow the practice gaps in Ethiopia.

\section{Abbreviations \\ AOR: Adjusted odds ratio \\ CI: Confidence interval \\ COR: Crude odds ratio \\ HIV: Human immuno virus \\ INH: Isoniazid \\ IPT: Isoniazid preventive therapy \\ PTB+: Smear-positive pulmonary tuberculosis \\ TB: Tuberculosis \\ SD: $\quad$ Standard deviation \\ SPSS: Statistical Package for the Social Sciences \\ WHO: World Health Organization.}

\section{Data Availability}

The datasets supporting the conclusions of this article are included within the article.

\section{Ethical Approval}

The institutional review board of Jimma University Institute of Health approved the study. A formal letter of support was written from the Institute of Health IRB to Amhara regional state administrator's office, and the procedures were in compliance with Helsinki Declaration.

\section{Conflicts of Interest}

The authors declare that they have no competing interests.

\section{Authors' Contributions}

YW, LA, NF, and AM contributed to the conceptualization, literature review, design, analysis, and writing of the paper. All authors read and approved the final submitted paper. The authors declare that they have agreed to publish on this journal.

\section{Acknowledgments}

We would like to acknowledge the assistance of Jimma University, Institute of Health, and Faculty of Public Health in undertaking this research. We would also like to express our gratitude to the study participants and officials working in Amhara regional state, Bahir Dar special zone administration, which really deserve acknowledgement for their cooperation. Last but not least, our thanks also go to the data collectors, supervisors, and advisors. This research was funded by Jimma University, Institute of Health, and Faculty of Public Health. 


\section{References}

[1] G. Tizazu and T. Anteneh, Internal medicine lecture notes for health officers. First edit. Ethiopia Public Health Training Initiative, The Carter Center, the Ethiopia Ministry of Health, and the Ethiopia Ministry of Education, 2006.

[2] WHO, Global tuberculosis report, World Health Organization, Geneva, 2020, https://www.who.int/news-room/fact-sheets/ detail/tuberculosis/2020.

[3] WHO, Global tuberculosis report, World Health Organization, Geneva, 2019, https://www.who.int/tb/publications/global_ report/en/.

[4] EFMoH, Annual performance report, Addis Ababa, Ethiopia, 2015.

[5] J. R. Starke, "Improving tuberculosis care for children in highburden settings," Pediatrics, vol. 134, no. 4, pp. 655-657, 2014.

[6] Health department of Republic of South of Africa, Guidelines for the management of tuberculosis in children, p. 8, 2014.

[7] Virginia department of Health, Screening for TB infection and disease in Virginia, Virginia Health Department, 2013.

[8] EFMoH, Guidelines for clinical and programmatic management of TB, leprosy and TB/HIV in Ethiopia, Ethiopian federal Ministery of Health, Addis Ababa, Fifth edition, 2012.

[9] R. Triasih, C. F. Robertson, T. Duke, and S. M. Graham, “A prospective evaluation of the symptom-based screening approach to the management of children who are contacts of tuberculosis cases," Clinical Infectious Diseases, vol. 60, no. 1, pp. 12-18, 2014.

[10] WHO, Guidance for national tuberculosis programmes on the management of tuberculosis in children, World Health Organization, 2014, https://www.who.int/tb/publications/childtb_ guidelines/en/.

[11] World Health Organization Stop TB Partnership Childhood TB Subgroup, "Childhood contact screening and management," The International Journal of Tuberculosis and Lung Disease, vol. 11, no. 1, pp. 12-15, 2007.

[12] CDC, "The use of preventive therapy for tuberculous infection in the United States. Recommendations of the Advisory Committee for Elimination of Tuberculosis," Morbidity and Mortality Weekly Report, vol. 39, no. 8, pp. 9-12, 1990.

[13] J. Ayieko, L. Abuogi, B. Simchowitz, E. A. Bukusi, A. H. Smith, and A. Reingold, "Efficacy of isoniazid prophylactic therapy in prevention of tuberculosis in children : a meta analysis," BMC Infectious Diseases, vol. 14, no. 1, pp. 1-10, 2014.

[14] S. S. van Wyk, A. J. Reid, A. M. Mandalakas et al., "Operational challenges in managing isoniazid preventive therapy in child contacts: a high-burden setting perspective," BMC Public Health, vol. 11, no. 1, pp. 1-6, 2011.

[15] Y. Tadesse, N. Gebre, S. Daba et al., "Uptake of isoniazid preventive therapy among under-five children : TB contact investigation as an entry point," PLoS One, vol. 11, no. 5, 2016.

[16] M. Bonnet, C. Kyakwera, N. Kyomugasho et al., "Prospective cohort study of the feasibility and yield of household child tuberculosis contact screening in Uganda," The International Journal of Tuberculosis and Lung Disease, vol. 21, no. 8, pp. 862-868, 2017.

[17] WHO, Country profiles for 22 hihg-burded countries, WHO, 2015.

[18] L. F. Assebe, H. L. Reda, A. D. Wubeneh, W. T. Lerebo, and S. M. Lambert, "The effect of isoniazid preventive therapy on incidence of tuberculosis among HIV-infected clients under pre-ART care, Jimma, Ethiopia: a retrospective cohort study," BMC Public Health, vol. 15, no. 1, 2015.

[19] World Health Organization, Gear up to end TB: introducing the end TB strategy, World Health Organization, 2015, https://apps.who.int/iris/handle/10665/156394.

[20] WHO, The end TB strategy, World Health Organization, Geneva, 2015, http://www.who.int/tb/End_TB_brochure .pdf?ua $=1$.

[21] H. R. Shivaramakrishna, A. Frederick, A. Shazia et al., "Isoniazid preventive treatment in children in two districts of South India: does practice follow policy?," The International Journal of Tuberculosis and Lung Disease, vol. 18, no. 8, pp. 919-924, 2014.

[22] G. B. Gebregergs and W. G. Alemu, "Household contact screening adherence among tuberculosis patients in northern Ethiopia," PLoS One, vol. 10, no. 5, 2015.

[23] M. Pothukuchi, S. B. Nagaraja, S. Kelamane et al., "Tuberculosis contact screening and isoniazid preventive therapy in a south Indian district : operational issues for programmatic consideration," PLoS One, vol. 6, no. 7, 2011.

[24] S. Jirapaiboonsuk and R. S. Chapman, "Knowledge, Attitude, and Practice Towards Childhood Tuberculosis in Guardians of Patients Visiting the Pediatric out-Patient Department, Sirindhorn Hospital, Bangkok," Journal of Health Research, vol. 24, no. 2, pp. 101-106, 2010.

[25] G. J. Fox, L. P. Loan, N. V. Nhung et al., "Barriers to adherence with tuberculosis contact investigation in six provinces of Vietnam: a nested case-control study," BMC Infectious Diseases, vol. 15, no. 1, pp. 1-8, 2015.

[26] N. J. M. Claessens, F. F. Gausi, S. Meijnen, M. M. Weismuller, F. M. Salaniponi, and A. D. Harries, "Screening childhood contacts of patients with smear-positive pulmonary tuberculosis in Malawi," The International Journal of Tuberculosis and Lung Disease, vol. 6, no. 4, pp. 362-364, 2002.

[27] S. Tornee, J. Kaewkungwal, W. Fungladda, U. Silachamroon, P. Akarasewi, and P. Sunakorn, "Factors associated with the household contact screening adherence of tuberculosis patients," Southeast Asian Journal of Tropical Medicine \& Public Health, vol. 36, no. 2, pp. 331-340, 2005.

[28] N. Beyers, R. P. Gie, H. S. Schaaf et al., "A prospective evaluation of children under the age of 5 years living in the same household as adults with recently diagnosed pulmonary tuberculosis, South Africa," The International Journal of Tuberculosis and Lung Disease, vol. 1, no. 1, pp. 38-43, 2007.

[29] M. E. Rutherford, R. Ruslami, M. Anselmo et al., "Management of children exposed to Mycobacterium tuberculosis: a public health evaluation in West Java, Indonesia," Bulletin of the World Health Organization, vol. 91, no. 12, pp. 932941A, 2013.

[30] V. V. Banu Rekha, K. Jagarajamma, F. Wares, V. Chandrasekaran, and S. Swaminathan, "Contact screening and chemoprophylaxis in India's Revised Tuberculosis Control Programme : a situational analysis," The International Journal of Tuberculosis and Lung Disease, vol. 13, no. 12, pp. 1507-1512, 2009.

[31] C. Hall, P. Sukijthamapan, R. dos Santos et al., "Challenges to delivery of isoniazid preventive therapy in a cohort of children exposed to tuberculosis in Timor-Leste," Tropical Medicine \& International Health, vol. 20, pp. 730-736, 2015. 
[32] A. R. Singh, A. Kharate, P. Bhat et al., "Isoniazid preventive therapy among children living with tuberculosis patients: is it working? A mixed-method study from Bhopal, India," Journal of Tropical Pediatrics, vol. 63, no. 4, pp. 274-285, 2017.

[33] M. Nyirenda, R. Sinfield, S. Haves, E. M. Molyneux, and S. M. Graham, "Poor attendance at a child TB contact clinic in Malawi," The International Journal of Tuberculosis and Lung Disease, vol. 10, no. 5, pp. 585-587, 2006. 